\title{
HDL and associated factors stratified by sex and menopausal status: results from a community-based survey in Taiwan
}

\author{
Huan-Cheng Chang ${ }^{1,2}$, Chuan-Fa Hsieh ${ }^{3,4}$, Disline Manli Tantoh ${ }^{5}$, Pei-Chieh Ko ${ }^{5}$, Ya- \\ Yu Kung, ${ }^{6}$ Mei-Chi Lin ${ }^{6}$, Yi-Ching Liaw ${ }^{7}$ and Yung-Po Liaw ${ }^{5,8}$ \\ ${ }^{1}$ Division of Family Medicine, Department of Community Medicine, Landseed Hospital, Taoyuan, Taiwan \\ ${ }^{2}$ Department of Health Care Management, Chang Gung University, Taoyuan, Taiwan \\ ${ }^{3}$ Department of Medical Education and Research, Landseed Hospital, Taoyuan, Taiwan \\ ${ }^{4}$ Center for General Education, Hsin Sheng College of Medical Care and Management, Taoyuan, Taiwan \\ ${ }^{5}$ Department of Public Health and Institute of Public Health, Chung Shan Medical University, Taichung, Taiwan \\ ${ }^{6}$ Division of Health Management, Landseed Hospital, Taoyuan, Taiwan \\ ${ }^{7}$ Institute of Clinical Medicine, National Yang-Ming University, Taipei, Taiwan \\ ${ }^{8}$ Department of Family and Community Medicine, Chung Shan Medical University, Taichung, Taiwan \\ Correspondence to: Yung-Po Liaw, email: Liawyp@csmu.edu.tw \\ Keywords: HDL; menopause; sex; factors; Taiwan
}

Received: January 10, $2018 \quad$ Accepted: February 27, $2018 \quad$ Published: March 27, 2018

Copyright: Chang et al. This is an open-access article distributed under the terms of the Creative Commons Attribution License 3.0 (CC BY 3.0), which permits unrestricted use, distribution, and reproduction in any medium, provided the original author and source are credited.

\section{ABSTRACT}

Aim: To investigate factors, especially modifiable factors associated with highdensity lipoprotein (HDL) in Taiwanese based on sex and menopausal status.

Materials and Methods: Participants comprised 2022 men and 2392 women (1267 menopausal and 1125 non-menopausal) aged $\geq 30$ years who resided in Pingzhen district, Taoyuan from 2006-2011. Their data, obtained through questionnaires and measurements were retrieved from the Li-Shin Hospital.

Results: Higher HDL was associated with total cholesterol, underweight, and alcohol drinking in both men and women. It was also associated with education, blood group B, and marital status in men as well as with age in women. Moreover, it was associated with total cholesterol, underweight, and age in both menopausal and non-menopausal women. Furthermore, it was associated with marital status in non-menopausal women and alcohol drinking in menopausal women. Lower HDL was associated with triglycerides, low-density lipoprotein (LDL), overweight, obesity, waist-hip ratio (WHR), uric acid, and smoking in both men and women and with coffee drinking in only women. It was also associated with uric acid, triglycerides, LDL, overweight, obesity, WHR, and body fat in both menopausal and non-menopausal women. Moreover, it was associated with coffee drinking in menopausal women.

Conclusion: Modifiable factors associated with HDL differ according to sex and menopausal status. Sex and menopausal status should be considered when implementing lifestyle changes to raise HDL. For example, both men and women should maintain a normal weight as well as quit smoking.

\section{INTRODUCTION}

HDL cholesterol is an important biomarker of health especially cardiovascular and metabolic health
[1]. A vital function of HDL is to enhance the reverse cholesterol transport (RCT) pathway which accounts for its anti-atherosclerotic property [2]. In this pathway, cholesterol is carried to the liver for biliary excretion 
thereby preventing its accumulation in the arterial wall. HDL's anti-inflammatory, anti-oxidative, anti-apoptotic, anti-infectious, and anti-thrombotic properties are also well known [1, 3-5]. Maintaining HDL at desired levels is of clinical significance. The beneficial minimal cutoff levels of HDL are $\geq 40$ and $\geq 50 \mathrm{mg} / \mathrm{dL}$ for men and women, respectively [6-8]. A desired HDL level (e.g., $>40 \mathrm{mg} / \mathrm{dL}$ ) is a vital therapeutic target in primary and secondary prevention [9]. It is believed that low levels of HDL need to be raised in many patients even if LDL or non-HDL levels are yet to be reduced to the target levels [10]. For instance, for individuals with low HDL and hypertriglyceridemia, metabolic syndrome, type 2 diabetes mellitus or a high cardiovascular risk $(>20 \%)$, reaching the HDL therapeutic target is recommended [9]. Because of the health impacts of HDL, it is important to study factors that influence it. A greater fraction of these factors is inherited [11-13]. However, socio-demographic and lifestyle factors including age, sex, menopausal status, hormone replacement therapy, exercise, alcohol drinking, BMI, smoking, and diet are non-genetic [11-16]. Lifestyle factors like exercise, alcohol drinking, smoking, BMI, and diet are modifiable and can raise HDL levels if well managed [15]. Raising HDL levels can in turn help in preventing cardiovascular diseases. There are some controversies regarding HDL and its associated factors especially when menopausal status is considered [16-19]. HDL levels have been shown to be significantly different between men and women $[17,20,21]$. This study was conducted to investigate factors, especially modifiable factors associated with HDL under stratification by sex and menopausal status.

\section{RESULTS}

Tables 1 and 2 show the participants characteristics based on sex and menopausal status, respectively. There was a significant difference $(P=0.0021)$ between the percentage of men and that of women as far as HDL is concerned (Table 1). However, the percentage of menopausal women was not significantly different from that of the non-menopausal women (Table 2). Tables 3 and 4 show the factors associated with HDL stratified by sex and menopausal status, respectively. Higher HDL was associated with total cholesterol, underweight, and alcohol drinking in both men and women. It was also associated with age 50-69 years, education, blood group $\mathrm{B}$, and marital status (single). However, the association with age was significant only in women while that with education, blood group B, and single status was significant only in men. Lower HDL was associated with uric acid, triglycerides, LDL $\geq 130 \mathrm{mg} / \mathrm{dL}$, BMI (overweight and obesity), waist-hip ratio, and smoking in both men and women and with coffee drinking only in women (Table 3). Based on menopausal status, higher HDL was associated with age (50-59 years), total cholesterol and BMI (underweight) in both menopausal and non-menopausal women (Table 4). In addition, it was associated with marital status (divorced and widowed) and alcohol drinking. However, the association with marital status was only in non-menopausal women while that with alcohol drinking was only in menopausal women (Table 4). Lower HDL was associated with uric acid, triglycerides, LDL $\geq 130 \mathrm{mg} / \mathrm{dL}, \mathrm{BMI}$ (overweight and obese), waist-hip ratio, and body fat in both menopausal and non-menopausal women. Moreover, it was associated with coffee drinking. However, this association was only in menopausal women (Table 4).

\section{DISCUSSION}

In the current study, factors associated with HDL were determined based on sex and menopausal status. Based on sex, there were significant differences between men and women as far as HDL is concerned. To our knowledge, this study is among the first in Taiwan to stratify the factors that are associated with HDL by sex and menopausal status. A study investigated the effects of gender and menopausal status on plasma lipoprotein subspecies and particle sizes [22]. However, its aim differs from that of the current study in terms of the outcome since this study's outcome was only HDL. Moreover, some previous studies conducted in women focused generally on cardiovascular risk factors and lipid profiles not specifically HDL [23-25]. It is well known that HDL levels in men and women significantly differ $[17,20,21$, 26]. One reason for this is the influence of estrogen and testosterone on the activities of hepatic lipase. Hepatic lipase plays a role in HDL metabolism and its levels are inversely related with those of HDL [27]. Estrogen and testosterone respectively tend to decrease and increase hepatic lipase levels $[26,28]$. As a result, women tend to have higher HDL levels than men $[17,20,21,26]$. In this study, the percentage of menopausal women was not significantly different from that of the non-menopausal women. Several studies on the association between HDL and menopausal status have shown controversial results [16-19, 29]. It is still unclear whether the relationship between HDL and menopausal status is due to hormone levels [30, 31]. However, estrogen is believed to be associated with higher HDL levels [28, 32]. At post menopause, androgen levels are higher than estrogen levels thereby leading to relatively lower levels of HDL [31, 32]. HDL, body fat, waist circumference, BMI, triglyceride, and LDL are known risk factors of metabolic syndrome [33, 34]. Triglyceride, LDL, higher BMI, increased waist circumference, and body fat have been associated with lower HDL while lower BMI and total cholesterol have been associated with higher HDL [11, 20, 26, 35-37]. Among the metabolic biomarkers mentioned above, total cholesterol, triglyceride, LDL, and waist-hip ratio were associated with $\mathrm{HDL}$ in both sexes and 
Table 1: Characteristics of male $(n=2,022)$ and female $(n=2,392)$ participants

\begin{tabular}{|c|c|c|c|}
\hline Variable & Men n(\%) & Women n(\%) & P-value \\
\hline HDL (mg/dL) & & & 0.0021 \\
\hline Male $\geq 40$; female $\geq 50$ & $1,769(87.49)$ & $2,015(84.24)$ & \\
\hline Male $<40$; female $<50$ & $253(12.51)$ & $377(15.76)$ & \\
\hline Total cholesterol (mg/dL) & & & 0.0001 \\
\hline$<200$ & $1,042(51.53)$ & $1,095(45.78)$ & \\
\hline$\geq 200$ & $980(48.47)$ & $1,297(54.22)$ & \\
\hline Triglycerides (mg/dL) & & & $<.0001$ \\
\hline$<150$ & $1,361(67.31)$ & $1,906(79.68)$ & \\
\hline$\geq 150$ & $661(32.69)$ & $486(20.32)$ & \\
\hline $\operatorname{LDL}(\mathrm{mg} / \mathrm{dL})$ & & & 0.3500 \\
\hline$<130$ & $1,168(57.76)$ & $1,415(59.16)$ & \\
\hline$\geq 130$ & $854(42.24)$ & $977(40.84)$ & \\
\hline BMI $\left(\mathrm{kg} / \mathrm{m}^{2}\right)$ & & & $<.0001$ \\
\hline BMI<18.5 (Underweight) & $45(2.23)$ & $84(3.51)$ & \\
\hline $18.5 \leq \mathrm{BMI}<24$ (Normal) & $780(38.58)$ & $1,226(51.25)$ & \\
\hline $24 \leq \mathrm{BMI}<27$ (Overweight) & 707(34.97) & $607(25.38)$ & \\
\hline $\mathrm{BMI} \geq 27$ (Obese) & $490(24.23)$ & $475(19.86)$ & \\
\hline Waist circumference $(\mathrm{cm})$ & & & 0.0252 \\
\hline Male $<90$; female $<80$ & $1,341(66.32)$ & $1,509(63.09)$ & \\
\hline Male $\geq 90 ;$ female $\geq 80$ & $681(33.68)$ & $883(36.91)$ & \\
\hline Waist-hip ratio & & & $<.0001$ \\
\hline Male $<0.9$; female $<0.8$ & $1,118(55.29)$ & $1,003(41.93)$ & \\
\hline Male $\geq 0.9$; female $\geq 0.8$ & $904(44.71)$ & $1,389(58.07)$ & \\
\hline Body fat $(\%)$ & & & $<.0001$ \\
\hline Male $<25$; female $<30$ & $1,213(59.99)$ & $936(39.13)$ & \\
\hline Male $\geq 25$; female $\geq 30$ & $809(40.01)$ & $1,456(60.87)$ & \\
\hline Age (years) & & & $<.0001$ \\
\hline $30-49$ & $811(40.11)$ & $1,099(45.94)$ & \\
\hline $50-69$ & $846(41.84)$ & $1,197(50.04)$ & \\
\hline$\geq 70$ & $365(18.05)$ & $96(4.01)$ & \\
\hline Education & & & $<.0001$ \\
\hline University and above & $619(30.61)$ & $410(17.14)$ & \\
\hline Senior high & $672(33.23)$ & $754(31.52)$ & \\
\hline Junior high & $320(15.83)$ & $439(18.35)$ & \\
\hline Elementary and below & $411(20.33)$ & $789(32.98)$ & \\
\hline
\end{tabular}

(Continued) 


\begin{tabular}{|c|c|c|c|}
\hline Variable & Men n(\%) & Women n(\%) & P-value \\
\hline Blood type & & & 0.3082 \\
\hline A & $531(26.26)$ & $637(26.63)$ & \\
\hline B & $466(23.05)$ & $519(21.70)$ & \\
\hline $\mathrm{O}$ & $901(44.56)$ & $1,112(46.49)$ & \\
\hline $\mathrm{AB}$ & $124(6.13)$ & $124(5.18)$ & \\
\hline Marital status & & & $<.0001$ \\
\hline Single & $126(6.23)$ & $72(3.01)$ & \\
\hline Married & $1,826(90.31)$ & $2,167(90.59)$ & \\
\hline Co-habiting & $5(0.25)$ & $8(0.33)$ & \\
\hline Divorced & $42(2.08)$ & $65(2.72)$ & \\
\hline Widowed & $22(1.09)$ & $79(3.30)$ & \\
\hline Others & $1(0.05)$ & $1(0.04)$ & \\
\hline Fasting blood glucose (mg/dL) & & & 0.2510 \\
\hline$<126$ & $1,884(93.18)$ & $2,249(94.02)$ & \\
\hline$\geq 126$ & $138(6.82)$ & $143(5.98)$ & \\
\hline Creatinine (mg/dL) & & & $<.0001$ \\
\hline$<1.4$ & $1,844(91.20)$ & $2,365(98.87)$ & \\
\hline$\geq 1.4$ & $178(8.80)$ & $27(1.13)$ & \\
\hline Uric acid (mg/dL) & & & $<.0001$ \\
\hline Male $\leq 7$; female $\leq 6$ & $1,351(66.82)$ & $1,948(81.44)$ & \\
\hline Male $>7$; female $>6$ & $671(33.18)$ & $444(18.56)$ & \\
\hline $\operatorname{AST}(\mathbf{U} / \mathbf{L})$ & & & $<.0001$ \\
\hline$<40$ & $1,848(91.39)$ & $2,260(94.48)$ & \\
\hline$\geq 40$ & $174(8.61)$ & $132(5.52)$ & \\
\hline ALT (U/L) & & & $<.0001$ \\
\hline$<40$ & $1,623(80.27)$ & $2,147(89.76)$ & \\
\hline$\geq 40$ & $399(19.73)$ & $245(10.24)$ & \\
\hline SBP (mmHg) & & & $<.0001$ \\
\hline$<120$ & $630(31.16)$ & $1,222(51.09)$ & \\
\hline $120-139$ & $856(42.33)$ & $749(31.31)$ & \\
\hline$\geq 140$ & $536(26.51)$ & $421(17.60)$ & \\
\hline DBP (mmHg) & & & $<.0001$ \\
\hline$<80$ & $1,114(55.09)$ & $1,687(70.53)$ & \\
\hline $80-89$ & $562(27.79)$ & $468(19.57)$ & \\
\hline$\geq 90$ & $346(17.11)$ & $237(9.91)$ & \\
\hline Smoking & & & $<.0001$ \\
\hline Never & $1,010(49.95)$ & $2,299(96.11)$ & \\
\hline Quit & 289(14.29) & $22(0.92)$ & \\
\hline Current & $723(35.76)$ & $71(2.97)$ & \\
\hline
\end{tabular}

(Continued) 


\begin{tabular}{|c|c|c|c|}
\hline Variable & Men n(\%) & Women n(\%) & P-value \\
\hline Alcohol drinking & & & $<.0001$ \\
\hline Never & $1,464(72.40)$ & $2,299(96.11)$ & \\
\hline Quit & $93(4.60)$ & $8(0.33)$ & \\
\hline Current & $465(23.00)$ & $85(3.55)$ & \\
\hline Betel nut chewing & & & $<.0001$ \\
\hline Never & $1,742(86.15)$ & $2,378(99.41)$ & \\
\hline Quit & $138(6.82)$ & $5(0.21)$ & \\
\hline Current & $142(7.02)$ & $9(0.38)$ & \\
\hline Exercise & & & 0.4399 \\
\hline No & $717(35.46)$ & $875(36.58)$ & \\
\hline Yes & $1,305(64.54)$ & $1,517(63.42)$ & \\
\hline Vegetarian & & & $<.0001$ \\
\hline No & $1,874(92.68)$ & $2,132(89.13)$ & \\
\hline Yes & $148(7.32)$ & $260(10.87)$ & \\
\hline Coffee drinking & & & 0.0075 \\
\hline$<3$ times per week & $370(18.30)$ & $515(21.53)$ & \\
\hline$\geq 3$ times per week & $1,652(81.70)$ & $1,877(78.47)$ & \\
\hline \multicolumn{4}{|l|}{ Disease history } \\
\hline Diabetes & $146(7.22)$ & $130(5.43)$ & 0.0146 \\
\hline Hypertension & $409(20.23)$ & $355(14.84)$ & $<.0001$ \\
\hline Heart disease & $123(6.08)$ & $92(3.85)$ & 0.0006 \\
\hline Hyperlipidemia & $147(7.27)$ & $119(4.97)$ & 0.0014 \\
\hline Stroke & $31(1.53)$ & $3(0.13)$ & $<.0001$ \\
\hline \multicolumn{4}{|l|}{ Menopause } \\
\hline No & & $1,125(47.03)$ & \\
\hline Yes & & $1,267(52.97)$ & \\
\hline \multicolumn{4}{|l|}{ Hormone therapy } \\
\hline No & & $2,145(89.67)$ & \\
\hline Yes & & $247(10.33)$ & \\
\hline \multicolumn{4}{|l|}{ Oral contraceptives } \\
\hline No & & $2,281(95.36)$ & \\
\hline Yes & & $111(4.64)$ & \\
\hline
\end{tabular}

menopausal statuses in this study. However, BMI and body fat did not yield similar results based on our stratification. For instance, the different BMI categories were associated with HDL in both men and women. That is, underweight was significantly associated with higher HDL while overweight and obesity were significantly associated with lower HDL in both men and women. Stratification by menopausal status yielded similar results. However, significant results were prominent only in non- menopausal women. It is unclear why significant associations were found only in non-menopausal women. Body fat was associated with lower HDL only in women. However, it was associated with lower HDL in both menopausal and non-menopausal women. Again, it is still unclear why there was no significant association in men. So far, the associations between age and HDL have not been consistent. For instance, in some studies, higher HDL levels were observed among older African Americans 
Table 2: Characteristics of non-menopausal $(n=1,125)$ and menopausal $(n=1,267)$ women

\begin{tabular}{|c|c|c|c|}
\hline Variable & No menopause $\mathbf{n}(\%)$ & Menopause n(\%) & P-value \\
\hline HDL (mg/dL) & & & 0.8829 \\
\hline$\geq 50$ & $949(84.36)$ & 1066(84.14) & \\
\hline$<50$ & $176(15.64)$ & 201(15.86) & \\
\hline Total cholesterol (mg/dL) & & & $<.0001$ \\
\hline$<200$ & $662(58.84)$ & $433(34.18)$ & \\
\hline$\geq 200$ & $463(41.16)$ & $834(65.82)$ & \\
\hline Triglycerides (mg/dL) & & & $<.0001$ \\
\hline$<150$ & $979(87.02)$ & $927(73.16)$ & \\
\hline$\geq 150$ & $146(12.98)$ & $340(26.84)$ & \\
\hline LDL (mg/dL) & & & $<.0001$ \\
\hline$<130$ & $810(72.00)$ & $605(47.75)$ & \\
\hline$\geq 130$ & $315(28.00)$ & $662(52.25)$ & \\
\hline BMI $\left(\mathrm{kg} / \mathrm{m}^{2}\right)$ & & & $<.0001$ \\
\hline $\mathrm{BMI}<18.5$ (Underweight) & $56(4.98)$ & $28(2.21)$ & \\
\hline $18.5 \leq \mathrm{BMI}<24$ (Normal) & $672(59.73)$ & $554(43.73)$ & \\
\hline 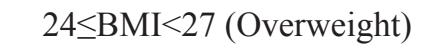 & $237(21.07)$ & $370(29.20)$ & \\
\hline BMI $\geq 27$ (Obese) & $160(14.22)$ & $315(24.86)$ & \\
\hline Waist circumference $(\mathrm{cm})$ & & & $<.0001$ \\
\hline$<80$ & $821(72.98)$ & $688(54.30)$ & \\
\hline$\geq 80$ & $304(27.02)$ & $579(45.70)$ & \\
\hline Waist-hip ratio & & & $<.0001$ \\
\hline$<0.8$ & $604(53.69)$ & 399(31.49) & \\
\hline$\geq 0.8$ & $521(46.31)$ & $868(68.51)$ & \\
\hline Body fat (\%) & & & $<.0001$ \\
\hline$<30$ & $536(47.64)$ & $400(31.57)$ & \\
\hline$\geq 30$ & $589(52.36)$ & $867(68.43)$ & \\
\hline Age (years) & & & $<.0001$ \\
\hline $30-49$ & $966(85.87)$ & $133(10.50)$ & \\
\hline $50-69$ & $159(14.13)$ & $1,038(81.93)$ & \\
\hline$\geq 70$ & $0(0.00)$ & $96(7.58)$ & \\
\hline Education & & & $<.0001$ \\
\hline University and above & $327(29.07)$ & $83(6.55)$ & \\
\hline Senior high & $512(45.51)$ & $242(19.10)$ & \\
\hline Junior high & $197(17.51)$ & $242(19.10)$ & \\
\hline Elementary and below & $89(7.91)$ & $700(55.25)$ & \\
\hline
\end{tabular}




\begin{tabular}{|c|c|c|c|}
\hline Variable & No menopause $n(\%)$ & Menopause n(\%) & P-value \\
\hline Blood type & & & 0.4452 \\
\hline A & $306(27.20)$ & $331(26.12)$ & \\
\hline B & $254(22.58)$ & $265(20.92)$ & \\
\hline $\mathrm{O}$ & $513(45.60)$ & $599(47.28)$ & \\
\hline $\mathrm{AB}$ & $52(4.62)$ & $72(5.68)$ & \\
\hline Marital status & & & $<.0001$ \\
\hline Single & $57(5.07)$ & $15(1.18)$ & \\
\hline Married & $1,018(90.49)$ & $1,149(90.69)$ & \\
\hline Co-habiting & $1(0.09)$ & $7(0.55)$ & \\
\hline Divorced & $39(3.47)$ & $26(2.05)$ & \\
\hline Widowed & $10(0.89)$ & $69(5.45)$ & \\
\hline Others & $0(0.00)$ & $1(0.08)$ & \\
\hline Fasting blood glucose $(\mathrm{mg} / \mathrm{dL})$ & & & $<.0001$ \\
\hline$<126$ & $1,094(97.24)$ & $1,155(91.16)$ & \\
\hline$\geq 126$ & $31(2.76)$ & $112(8.84)$ & \\
\hline Creatinine (mg/dL) & & & 0.0007 \\
\hline$<1.4$ & $1,121(99.64)$ & $1,244(98.18)$ & \\
\hline$\geq 1.4$ & $4(0.36)$ & $23(1.82)$ & \\
\hline Uric acid (mg/dL) & & & $<.0001$ \\
\hline$\leq 6$ & $1,001(88.98)$ & $947(74.74)$ & \\
\hline$>6$ & $124(11.02)$ & $320(25.26)$ & \\
\hline $\operatorname{AST}(\mathrm{U} / \mathrm{L})$ & & & $<.0001$ \\
\hline$<40$ & $1,086(96.53)$ & $1,174(92.66)$ & \\
\hline$\geq 40$ & $39(3.47)$ & $93(7.34)$ & \\
\hline ALT (U/L) & & & $<.0001$ \\
\hline$<40$ & $1,042(92.62)$ & $1,105(87.21)$ & \\
\hline$\geq 40$ & $83(7.38)$ & $162(12.79)$ & \\
\hline SBP (mmHg) & & & $<.0001$ \\
\hline$<120$ & $733(65.16)$ & $489(38.60)$ & \\
\hline $120-139$ & $300(26.67)$ & $449(35.44)$ & \\
\hline$\geq 140$ & $92(8.18)$ & $329(25.97)$ & \\
\hline DBP (mmHg) & & & $<.0001$ \\
\hline$<80$ & $863(76.71)$ & $824(65.04)$ & \\
\hline $80-89$ & 181(16.09) & $287(22.65)$ & \\
\hline$\geq 90$ & $81(7.20)$ & $156(12.31)$ & \\
\hline Smoking & & & 0.0005 \\
\hline Never & $1,065(94.67)$ & $1,234(97.40)$ & \\
\hline Quit & $18(1.60)$ & $4(0.32)$ & \\
\hline Current & $42(3.73)$ & $29(2.29)$ & \\
\hline
\end{tabular}

(Continued) 


\begin{tabular}{|c|c|c|c|}
\hline Variable & No menopause $n(\%)$ & Menopause n(\%) & P-value \\
\hline Alcohol drinking & & & 0.1191 \\
\hline Never & $1,073(95.38)$ & $1,226(96.76)$ & \\
\hline Quit & $3(0.27)$ & $5(0.39)$ & \\
\hline Current & $49(4.36)$ & $36(2.84)$ & \\
\hline Betel nut chewing & & & 0.2393 \\
\hline Never & $1,118(99.38)$ & $1,260(99.45)$ & \\
\hline Quit & $4(0.36)$ & $1(0.08)$ & \\
\hline Current & $3(0.27)$ & $6(0.47)$ & \\
\hline Exercise & & & $<.0001$ \\
\hline No & $464(41.24)$ & $411(32.44)$ & \\
\hline Yes & $661(58.76)$ & $856(67.56)$ & \\
\hline Vegetarian & & & 0.0804 \\
\hline No & $1,016(90.31)$ & $1,116(88.08)$ & \\
\hline Yes & $109(9.69)$ & $151(11.92)$ & \\
\hline Coffee drinking & & & $<.0001$ \\
\hline$<3$ times per week & $321(28.53)$ & $194(15.31)$ & \\
\hline$\geq 3$ times per week & $804(71.47)$ & $1,073(84.69)$ & \\
\hline \multicolumn{4}{|l|}{ Disease history } \\
\hline Diabetes & $21(1.87)$ & $109(8.60)$ & $<.0001$ \\
\hline Hypertension & $65(5.78)$ & $290(22.89)$ & $<.0001$ \\
\hline Heart disease & $25(2.22)$ & $67(5.29)$ & $<.0001$ \\
\hline Hyperlipidemia & $32(2.84)$ & $87(6.87)$ & $<.0001$ \\
\hline Stroke & $2(0.18)$ & $1(0.08)$ & 0.4954 \\
\hline Hormone therapy & & & $<.0001$ \\
\hline No & $1,086(96.53)$ & $1,059(83.58)$ & \\
\hline Yes & $39(3.47)$ & $208(16.42)$ & \\
\hline Oral contraceptives & & & 0.3107 \\
\hline No & $1,078(95.82)$ & $1,203(94.95)$ & \\
\hline Yes & $47(4.18)$ & $64(5.05)$ & \\
\hline
\end{tabular}

especially females [38] and Chinese adults [39]. However, in another study, a significant association between age and higher HDL was prominent only among Hong Kong Chinese females [40]. In this study, the association between HDL and age was stronger in women (higher $\beta$ ) than men in all age groups. However, a significant association was found only in women aged 50-69 years. A study showed higher levels of HDL in those aged 60-69 years [21]. The prominent association between age and HDL only in women might be due to estrogen. However, at age 50-69 years, estrogen levels are believed to be relatively lower than those at younger ages. Therefore, this association might not be explained in terms of estrogen levels. This greater influence might have been due to higher baseline HDL in females. In a study, educational level was associated with lower HDL in men, though not in a significant manner [26]. However, in another study, it was significantly associated with higher HDL [41]. In the current study, all educational levels were associated with higher HDL levels in the male participants. The impact of education on HDL seems to be in an indirect manner. It is thought that education affects the modifiable factors like smoking, drinking, and exercise which in turn affect HDL levels [26, 41]. Based on this knowledge, the existence of 
Table 3: Linear regression analysis showing factors associated with HDL concentration based on sex

\begin{tabular}{|c|c|c|c|c|}
\hline & \multicolumn{2}{|c|}{ Men } & \multicolumn{2}{|c|}{ Women } \\
\hline & $\beta$ & P-value & $\beta$ & P-value \\
\hline Total cholesterol (Ref: <200) & - & - & - & - \\
\hline$\geq 200$ & 11.599 & $<.0001$ & 14.641 & $<.0001$ \\
\hline \multicolumn{5}{|l|}{ Triglycerides (Ref: <150) } \\
\hline$\geq 150$ & -9.518 & $<.0001$ & -12.307 & $<.0001$ \\
\hline LDL (Ref: <130) & - & - & - & - \\
\hline$\geq 130$ & -7.642 & $<.0001$ & -8.701 & $<.0001$ \\
\hline BMI (Ref: Normal) & - & - & - & - \\
\hline Underweight & 9.273 & $<.0001$ & 5.161 & 0.0003 \\
\hline Overweight & -2.849 & $<.0001$ & -2.583 & 0.0009 \\
\hline Obese & -4.337 & $<.0001$ & -2.925 & 0.0032 \\
\hline WHR (Ref: Male $<0.9$ and female $<0.8$ ) & - & - & - & - \\
\hline Male $\geq 0.9$ and female $\geq 0.8$ & -1.657 & 0.0092 & -3.016 & $<.0001$ \\
\hline \multicolumn{5}{|l|}{ Body fat (Ref: Male $<25$ and female $<30$ ) } \\
\hline Male $\geq 25$ and female $\geq 30$ & -1.167 & 0.0743 & -2.788 & $<.0001$ \\
\hline Age (Ref: 30-49) & - & - & - & - \\
\hline $50-69$ & 0.207 & 0.7470 & 2.459 & 0.0035 \\
\hline$\geq 70$ & 0.872 & 0.3569 & 2.775 & 0.0874 \\
\hline Education (Ref: University and above) & - & - & - & - \\
\hline Senior high & 2.025 & 0.0018 & -0.207 & 0.7919 \\
\hline Junior high & 1.788 & 0.0291 & 0.131 & 0.8857 \\
\hline Elementary and below & 1.661 & 0.0444 & 0.647 & 0.4920 \\
\hline Blood type (Ref: A) & - & - & - & - \\
\hline $\mathrm{B}$ & 1.590 & 0.0270 & 0.937 & 0.2041 \\
\hline $\mathrm{O}$ & -0.245 & 0.6930 & 0.219 & 0.7236 \\
\hline $\mathrm{AB}$ & 1.048 & 0.3518 & -0.129 & 0.9158 \\
\hline Marital status (Ref: Married) & - & - & - & - \\
\hline Single & 2.333 & 0.0291 & -0.351 & 0.8189 \\
\hline Co-habiting & 1.742 & 0.7301 & -4.630 & 0.2946 \\
\hline Divorced & 1.359 & 0.4466 & 2.803 & 0.0772 \\
\hline Widowed & -1.891 & 0.4373 & -1.008 & 0.4888 \\
\hline Others & -12.073 & 0.2891 & -8.667 & 0.4870 \\
\hline Creatinine (Ref: $<1.4)$ & - & - & - & - \\
\hline$\geq 1.4$ & -2.505 & 0.0096 & -1.997 & 0.4247 \\
\hline Uric acid (Ref: Male $\leq 7$ and female $\leq 6$ ) & - & - & - & - \\
\hline Male $>7$ and female $>6$ & -2.020 & 0.0004 & -2.784 & $<.0001$ \\
\hline
\end{tabular}

(Continued) 


\begin{tabular}{lccccc}
\hline & \multicolumn{2}{c}{ Men } & & \multicolumn{2}{c}{ Women } \\
\cline { 2 - 3 } \cline { 5 - 6 } & $\boldsymbol{\beta}$ & P-value & & $\boldsymbol{\beta}$ & P-value \\
\hline Smoking (Ref: Never) & - & - & & - & - \\
$\quad$ Quit & 1.578 & 0.0423 & & -0.137 & 0.9605 \\
$\quad$ Current & -1.591 & 0.0087 & & -3.247 & 0.0393 \\
Alcohol drinking (Ref: Never) & - & - & & - & - \\
$\quad$ Quit & 1.560 & 0.2098 & & 5.099 & 0.2650 \\
$\quad$ Current & 3.695 & $<.0001$ & & 3.355 & 0.0204 \\
$\quad$ Vegetarian (Ref: No) & - & - & & - & - \\
$\quad$ Yes & -1.071 & 0.2676 & & -2.038 & 0.0136 \\
Coffee drinking (Ref: $\geq 3$ times per week) & - & - & & - & - \\
$\quad<3$ times per week & 0.931 & 0.1627 & & -1.823 & 0.0043 \\
\hline
\end{tabular}

Adjusted for fasting blood glucose, GOT, GPT, SBP, DBP, betel nut chewing, exercise, diabetes, hypertension, heart disease, hyperlipidemia, stroke, menopausal status, hormone therapy, and oral contraceptives.

a significant association only in men can be explained. For instance, there was a positive association between HDL and quitting smoking in men only (Table 3). Moreover, the association between alcohol drinking and HDL was stronger in men than women (Table 3). Non-O blood groups have been associated with an increased risk of cardiovascular diseases $[42,43]$. On the other hand, all blood groups have been associated with low levels of HDL and therefore, higher cardiovascular risk profiles [44]. In contrast, only blood group B was associated with higher HDL in this study specifically among men. It is not certain if this might have been due to some genetic factors. Therefore, future studies should elaborately investigate this. In this study, higher HDL was associated with marital status (for single men as well as divorced and widowed non-menopausal women). In a previous study, HDL was higher in single than married men and women but statistical significance was achieved only in men [45]. In another study, there was an association between HDL and marital status [11]. Similar to our results, statistical significance was prominent only among single men. Furthermore, in another study, the HDL of married women was not significantly different from that of the divorced or widowed women [46]. It is not very clear why a significant association was prominent only in single men in the current study. However, this might partly be due to some modifiable factors like BMI which can be managed by physical activity. Single men are more likely to be more physically active than single women. This can help in lowering the BMI as well as increasing HDL levels. Furthermore, it is not very clear why significant associations were prominent only in divorced and widowed non-menopausal women in the current study. One of the reasons could be that non-menopausal females are likely to have higher HDL levels at baseline when compared to menopausal females due to differences in estrogen levels. Moreover, most of the menopausal females are younger and can also engage in more exercise relative to the menopausal women. Similar to the current study, creatinine and uric acid have been significantly associated with lower HDL (29-31). As previously shown, cigarette smoking is a well-known risk factor for low HDL $[7,11,14,16,40]$. However, moderate alcohol consumption is associated with higher $\operatorname{HDL}[8,11,15,20$, $37,40,47]$. Similarly, alcohol consumption was associated with higher HDL among both sexes. Based on menopausal status, a significant association was observed only in nonmenopausal females. The reason for the absence of a prominent association in menopausal females cannot be explicitly stated. Despite the positive association of alcohol with HDL, only moderate amounts should be consumed due to other health issues associated with alcohol drinking. Like our results, a meta-analysis of observational studies and clinical trials showed that a plant-based vegetarian diet reduced HDL cholesterol [48]. However, another meta-analysis showed no significant association between HDL and vegetarian diet [49]. Coffee drinking was shown to enhance the HDL mediated reverse cholesterol transport [50]. One of the reasons for cholesterol efflux from macrophages is the presence of phenolic acids in coffee [50]. However, coffee also contains kahweol and cafestol which are believed to increase cholesterol. In this study, coffee consumption was associated with decreased HDL in only women and this has previously been shown [37]. Stratification by menopausal status yielded similar results. However, this was only in non-menopausal women. The reason why the association between HDL and coffee was significant only in women, especially non-menopausal women is unknown. Our study was limited in its cross-sectional nature. 
Table 4: Linear regression analysis showing factors associated with HDL concentration based on menopausal status

\begin{tabular}{|c|c|c|c|c|}
\hline & \multicolumn{2}{|c|}{ No menopause } & \multicolumn{2}{|c|}{ Menopause } \\
\hline & $\beta$ & P-value & $\beta$ & P-value \\
\hline Total cholesterol (Ref: $<\mathbf{2 0 0}$ ) & - & - & - & - \\
\hline$\geq 200$ & 15.759 & $<.0001$ & 13.379 & $<.0001$ \\
\hline Triglycerides (Ref: <150) & - & - & - & - \\
\hline$\geq 150$ & -11.907 & $<.0001$ & -12.268 & $<.0001$ \\
\hline LDL (Ref: < 130) & - & - & - & - \\
\hline$\geq 130$ & -9.975 & $<.0001$ & -7.494 & $<.0001$ \\
\hline BMI (Ref: Normal) & - & - & - & - \\
\hline Underweight & 5.274 & 0.0032 & 4.569 & 0.0627 \\
\hline Overweight & -3.386 & 0.0041 & -2.024 & 0.0553 \\
\hline Obese & -5.383 & 0.0006 & -1.546 & 0.234 \\
\hline WHR (Ref: $<0.8$ ) & - & - & - & - \\
\hline$\geq 0.8$ & -2.273 & 0.0144 & -3.697 & $<.0001$ \\
\hline Body fat (Ref: <30) & - & - & - & - \\
\hline$\geq 30$ & -3.169 & 0.0016 & -2.347 & 0.0202 \\
\hline Age (Ref: 30-49) & - & - & - & - \\
\hline $50-69$ & 2.461 & 0.0404 & 2.522 & 0.0388 \\
\hline$\geq 70$ & - & - & 3.385 & 0.0680 \\
\hline Education (Ref: University and above) & - & - & - & - \\
\hline Senior high & -0.145 & 0.8741 & -0.219 & 0.8917 \\
\hline Junior high & 1.742 & 0.1461 & -1.906 & 0.2343 \\
\hline Elementary and below & -0.880 & 0.5909 & 0.274 & 0.8561 \\
\hline Blood type (Ref: A) & - & - & - & - \\
\hline $\mathrm{B}$ & 1.334 & 0.2097 & 0.794 & 0.4432 \\
\hline $\mathrm{O}$ & 1.575 & 0.0825 & -1.127 & 0.1898 \\
\hline $\mathrm{AB}$ & 1.017 & 0.5870 & -1.120 & 0.4892 \\
\hline Marital status (Ref: Married) & - & - & - & - \\
\hline Single & 0.328 & 0.8514 & -1.937 & 0.5540 \\
\hline Co-habiting & 12.639 & 0.3111 & -7.118 & 0.1331 \\
\hline Divorced & 4.194 & 0.0462 & 0.296 & 0.9061 \\
\hline Widowed & 9.034 & 0.0262 & -2.491 & 0.1137 \\
\hline Others & - & - & -9.490 & 0.4486 \\
\hline Creatinine (Ref: $<1.4)$ & - & - & - & - \\
\hline$\geq 1.4$ & 5.574 & 0.4177 & -2.444 & 0.3681 \\
\hline Uric acid (Ref: $\leq 6)$ & - & - & - & - \\
\hline$>6$ & -3.525 & 0.0049 & -2.620 & 0.0031 \\
\hline
\end{tabular}




\begin{tabular}{|c|c|c|c|c|}
\hline & \multicolumn{2}{|c|}{ No menopause } & \multicolumn{2}{|c|}{ Menopause } \\
\hline & $\bar{\beta}$ & P-value & $\bar{\beta}$ & P-value \\
\hline Smoking (Ref: Never) & - & - & - & - \\
\hline Quit & 0.466 & 0.8815 & -4.770 & 0.4599 \\
\hline Current & -3.605 & 0.0759 & -2.491 & 0.3288 \\
\hline Alcohol drinking (Ref: Never) & - & - & - & - \\
\hline Quit & 8.360 & 0.2864 & 1.784 & 0.7611 \\
\hline Current & 2.909 & 0.1267 & 4.565 & 0.0436 \\
\hline Vegetarian (Ref: No) & - & - & - & - \\
\hline Yes & -2.320 & 0.0674 & -2.024 & 0.0659 \\
\hline $\begin{array}{l}\text { Coffee drinking (Ref: } \geq 3 \text { times per } \\
\text { week) }\end{array}$ & - & - & - & - \\
\hline$<3$ times per week & -0.969 & 0.2499 & -2.987 & 0.0028 \\
\hline
\end{tabular}

Adjusted for fasting blood glucose, GOT, GPT, SBP, DBP, betel nut chewing, exercise, diabetes, hypertension, heart disease, hyperlipidemia, stroke, hormone therapy, and oral contraceptives.

\section{MATERIALS AND METHODS}

This study included 4414 participants comprising 2022 men and 2392 women who were 30 years and above. Of the female participants, 1267 were menopausal while 1125 were non-menopausal. The participants resided in the Pingzhen district of Taoyuan city from 2006 to 2011. Their data were retrieved from the Li-Shin Hospital, a regional hospital in Northern Taiwan. These data included age, education, blood type, marital status, fasting blood glucose, creatinine, uric acid, total cholesterol, triglycerides, HDL, LDL, aspartate transaminase (AST), alanine transaminase (ALT), systolic blood pressure (SBP), diastolic blood pressure (DBP), BMI, waist circumference, waist-hip ratio, body fat, smoking, alcohol drinking, betel nut chewing, exercise, vegetarian diet, coffee drinking, disease history, menopausal status, hormone therapy, and oral contraceptives. Chi-square test was used to compare the percentage of men with women as well that of menopausal with non-menopausal women. Multiple linear regression analysis was used to determine the relationship between HDL and the other variables. All statistical analyses were performed with SAS 9.4 (SAS Institute, Cary, NC, USA). This study was approved by the Antai Medical Care Cooperation Antai Tian-Sheng Memorial Hospital Institutional Review Board (No. 16-006-C0).

\section{CONCLUSIONS}

Modifiable factors associated with HDL differ according to sex and menopausal status. For example, LDL, triglycerides, and WHR were associated with HDL in both sexes and menopausal statuses. BMI, drinking, and smoking were associated with HDL in both sexes but not in both menopausal statuses. Vegetarian diet and coffee drinking were associated with HDL only in women but not in both menopausal statuses. Sex and menopausal status should be considered when implementing lifestyle changes to raise HDL. For example, to achieve desirable levels of HDL, it is vital for both men and women to maintain a normal weight as well as quit smoking.

\section{Author contributions}

$\mathrm{H}-\mathrm{CC}, \mathrm{C}-\mathrm{FH}$, and Y-PL conceived the experiments. $\mathrm{P}-\mathrm{CK}$ and Y-PL performed data management and statistical analysis. H-CC, DMT, Y-YK, M-CL, Y-CL, and Y-PL interpreted the data as well as drafted the manuscript. $\mathrm{H}-\mathrm{CC}$, DMT, and Y-PL critically reviewed the manuscript for intellectual contents. All listed authors helped revise the final manuscript.

\section{CONFLICTS OF INTEREST}

The authors declare no competing financial interest.

\section{FUNDING}

This work was funded by grants from the collaboration projects between Chung Shan Medical University and Landseed Hospital (CSMU-LSH-103-02, 104-01 and 105-01).

\section{REFERENCES}

1. Karathanasis SK, Freeman LA, Gordon SM, Remaley AT. The Changing Face of HDL and the Best Way to Measure It. Clin Chem. 2017; 63:196-210. 
2. Das B, Mishra T. Role of HDL-C in health and disease. JIACM. 2012; 13: 218-22.

3. Zhou L, Li C, Gao L, Wang A. High-density lipoprotein synthesis and metabolism. Mol Med Rep. 2015; 12:4015-21.

4. Murphy AJ. High density lipoprotein: assembly, structure, cargo, and functions. ISRN Physiol. 2013.

5. Mineo C, Shaul PW. Novel biological functions of high-density lipoprotein cholesterol. Circ Res. 2012; 111:1079-90.

6. Silva RC, Diniz Mde F, Alvim S, Vidigal PG, Fedeli LM, Barreto SM. Physical activity and lipid profile in the ELSABrasil study. Arq Bras Cardiol. 2016; 107:10-9.

7. Quispe R, Manalac RJ, Faridi KF, Blaha MJ, Toth PP, Kulkarni KR, Nasir K, Virani SS, Banach M, Blumenthal RS. Relationship of the triglyceride to high-density lipoprotein cholesterol (TG/HDL-C) ratio to the remainder of the lipid profile: The Very Large Database of Lipids-4 (VLDL-4) study. Atherosclerosis. 2015; 242:243-50.

8. Ouyang F, Kumar R, Pongracic J, Story RE, Liu X, Wang B, Xing H, Liu X, Li Z, Zhang W. Adiposity, serum lipid levels, and allergic sensitization in Chinese men and women. J Allergy Clin Immunol. 2009; 123:940-8. e10.

9. Ascaso JF, Fernández-Cruz A, Santos PG, Mijares AH, Rojas AM, Millán J, Pallardo LF, Pedro-Botet J, Pérez-Jiménez F, Pía G. Significance of High Density Lipoprotein-Cholesterol in Cardiovascular Risk Prevention. Am J Cardiovasc Drugs. 2004; 4:299-314.

10. Assmann G, Gotto AM. HDL cholesterol and protective factors in atherosclerosis. Circulation. 2004; 109:III8-14.

11. Kim SM, Han JH, Park HS. Prevalence of low HDLcholesterol levels and associated factors among Koreans. Circ J. 2006; 70:820-6.

12. Weissglas-Volkov D, Pajukanta P. Genetic causes of high and low serum HDL-cholesterol. J Lipid Res. 2010; 51:2032-57.

13. Azizi F. Genes associated with low serum high-density lipoprotein cholesterol. Arch Iran Med. 2014; 17:444.

14. Gossett LK, Johnson HM, Piper ME, Fiore MC, Baker TB, Stein JH. Smoking intensity and lipoprotein abnormalities in active smokers. J Clin Lipidol. 2009; 3:372-8.

15. Ellison RC, Zhang Y, Qureshi MM, Knox S, Arnett DK, Province MA. Lifestyle determinants of high-density lipoprotein cholesterol: The national heart, lung, and blood institute family heart study. Am Heart J. 2004; 147:529-35.

16. Fernandez ML, Murillo AG. (2016). Postmenopausal Women Have Higher HDL and Decreased Incidence of Low HDL than Premenopausal Women with Metabolic Syndrome. Healthcare: Multidisciplinary Digital Publishing Institute). Healthcare (Basel). 2016; 4:20.

17. Anagnostis P, Stevenson JC, Crook D, Johnston DG, Godsland IF. Effects of menopause, gender and age on lipids and high-density lipoprotein cholesterol subfractions. Maturitas. 2015; 81:62-8.
18. Polotsky HN, Polotsky AJ. Metabolic implications of menopause. Semin Reprod Med. 2010; 28:426-34.

19. Zhou JL, Lin SQ, Shen Y, Chen Y, Zhang Y, Chen FL. Serum lipid profile changes during the menopausal transition in Chinese women: a community-based cohort study. Menopause. 2010; 17:997-1003.

20. Ge P, Dong C, Ren X, Weiderpass E, Zhang C, Fan H, Zhang J, Zhang Y, Xi J. The high prevalence of low HDLcholesterol levels and dyslipidemia in rural populations in northwestern China. PloS One. 2015; 10:e144104.

21. Marhoum TA, Abdrabo AA, Lutfi MF. Effects of age and gender on serum lipid profile in over 55 years-old apparently healthy Sudanese individuals. AJBPS. 2013; 3:10.

22. Li Z, McNamara JR, Fruchart JC, Luc G, Bard JM, Ordovas J, Wilson P, Schaefer E. Effects of gender and menopausal status on plasma lipoprotein subspecies and particle sizes. J Lipid Res. 1996; 37:1886-96.

23. Pardhe BD, Ghimire S, Shakya J, Pathak S, Shakya S, Bhetwal A, Khanal PR, Parajuli NP. Elevated Cardiovascular Risks among Postmenopausal Women: A Community Based Case Control Study from Nepal. Biochem Res Int. 2017; 2017:3824903.

24. Torng PL, Su TC, Sung FC, Chien KL, Huang SC, Chow SN, Lee YT. Effects of menopause and obesity on lipid profiles in middle-aged Taiwanese women: the Chin-Shan Community Cardiovascular Cohort Study. Atherosclerosis. 2000; 153:413-21.

25. Ramezani Tehrani F, Behboudi-Gandevani S, Ghanbarian A, Azizi F. Effect of menopause on cardiovascular disease and its risk factors: a 9-year follow-up study. Climacteric. 2014; 17:164-72.

26. Kim HJ, Park HA, Cho YG, Kang JH, Kim KW, Kang JH, Kim NR, Chung WC, Kim CH, Whang DH. Gender difference in the level of HDL cholesterol in Korean adults. Korean J Fam Med. 2011; 32:173-81.

27. Jansen H, Verhoeven AJ, Sijbrands EJ. Hepatic lipase a pro-or anti-atherogenic protein? J Lipid Res. 2002; 43:1352-62.

28. Vaidya D. Sex hormones and circulating lipoprotein levels. Future Lipidology. 2008; 3:603-606.

29. Eapen DJ, Kalra GL, Rifai L, Eapen CA, Merchant N, Khan BV. Raising HDL cholesterol in women. Int J Womens Health. 2009; 1:181.

30. Al-Dahhan FH, Al-Naama LM, Disher A. Lipid profile and menopausal status. Al-Kindy Col Med. 2008; 4:8-19.

31. Weinberg ME, Manson JE, Buring JE, Cook NR, Seely EW, Ridker PM, Rexrode KM. Low sex hormone-binding globulin is associated with the metabolic syndrome in postmenopausal women. Metabolism. 2006; 55:1473-80.

32. Marchi R, Dell'Agnolo CM, Lopes TCR, Gravena AAF, Demitto MO, Brischiliari SCR, Borghesan DHP, Carvalho MDB, Pelloso SM. Prevalence of metabolic syndrome in pre-and postmenopausal women. Archives of Endocrinology and Metabolism. 2017; 61:160-166. 
33. Miller M, Stone NJ, Ballantyne C, Bittner V, Criqui MH, Ginsberg HN, Goldberg AC, Howard WJ, Jacobson MS, Kris-Etherton PM. Triglycerides and cardiovascular disease. Circulation. 2011; 123:2292-333.

34. Kwon H, Kim D, Kim JS. Body Fat Distribution and the Risk of Incident Metabolic Syndrome: A Longitudinal Cohort Study. Sci Rep. 2017; 7:10955.

35. Shamai L, Lurix E, Shen M, Novaro GM, Szomstein S, Rosenthal R, Hernandez AV, Asher CR. Association of body mass index and lipid profiles: evaluation of a broad spectrum of body mass index patients including the morbidly obese. Obes Surg. 2011; 21:42-7.

36. Ali ZA, Al-Zaidi MS. The association between body mass index, lipid profile and serum estradiol levels in a sample of iraqi diabetic premenopausal women. Oman Med J. 2011; 26:263.

37. Balk L, Hoekstra T, Twisk J. Relationship between longterm coffee consumption and components of the metabolic syndrome: the Amsterdam Growth and Health Longitudinal Study. Eur J Epidemiol. 2009; 24:203-9.

38. Harman JL, Griswold ME, Jeffries NO, Sumner AE, Sarpong DF, Akylbekova EL, Walker ER, Wyatt SB, Taylor Jr HA. Age is positively associated with HDL-cholesterol among African Americans in cross-sectional analysis: the Jackson Heart Study. J Clin Lipidol. 2011; 5:173.

39. Zuo H, Shi Z, Hu X, Wu M, Guo Z, Hussain A. Prevalence of metabolic syndrome and factors associated with its components in Chinese adults. Metabolism. 2009; 58:1102-8.

40. Cheung BM, Li M, Ong KL, Wat N, Tam S, Pang RW, Thomas GN, Woo J, Janus ED, Lau CP. High density lipoprotein-cholesterol levels increase with age in American women but not in Hong Kong Chinese women. Clin Endocrinol. 2009; 70:561-8.

41. Muennig P, Sohler N, Mahato B. Socioeconomic status as an independent predictor of physiological biomarkers of cardiovascular disease: evidence from NHANES. Prev Med. 2007; 45:35-40.

42. He M, Wolpin B, Rexrode K, Manson JE, Rimm E, Hu FB, Qi L. ABO blood group and risk of coronary heart disease in two prospective cohort studies. Arterioscler Thromb Vasc Biol. 2012; 32:2314-20.

43. Tanis B, Algra A, Van Der Graaf Y, Helmerhorst F, Rosendaal F. Procoagulant factors and the risk of myocardial infarction in young women. Eur J Haematol. 2006; 77:67-73.

44. Bartimaeus E, Waribo H. Relationship between ABO Blood Groups and Lipid Profile Level in Healthy Adult Residents in Port Harcourt Metropolis, Nigeria. JASEM. 2017; 21:1003-11.

45. Mirmiran P, Mohammadi F, Allahverdian S, Azizi F. Association of educational level and marital status with dietary intake and cardiovascular risk factors in Tehranian adults: Tehran lipid and glucose study (TLGS). Nutr Res. 2002; 22:1365-75.

46. Hosseinpour-Niazi S, Mirmiran P, Hosseinpanah F, Fallahghohroudy A, Azizi F. Association of marital status and marital transition with metabolic syndrome: tehran lipid and glucose study. Int J Endocrinol Metab. 2014; 12.

47. Jan CF, Chang HC, Tantoh DM, Chen PH, Liu WH, Huang JY, Wu MC, Liaw YP. Duration-response association between exercise and HDL in both male and female Taiwanese adults aged 40 years and above. Oncotarget. 2018; 9:2120-2127. https://doi.org/10.18632/ oncotarget.23251.

48. Yokoyama Y, Levin SM, Barnard ND. Association between plant-based diets and plasma lipids: a systematic review and meta-analysis. Nutr Rev. 2017; 75:683-98.

49. Zhang Z, Wang J, Chen S, Wei Z, Li Z, Zhao S, Lu W. Comparison of vegetarian diets and omnivorous diets on plasma level of HDL-c: a meta-analysis. PloS One. 2014; 9:e92609.

50. Uto-Kondo H, Ayaori M, Ogura M, Nakaya K, Ito M, Suzuki A, Takiguchi S, Yakushiji E, Terao Y, Ozasa H. Coffee consumption enhances high-density lipoproteinmediated cholesterol efflux in macrophages. Circ Res. 2010; 106:779-87. 